\title{
A Novel PSNR-B Approach for Evaluating the Quality of De-blocked Images
}

\author{
Trinadh Tadala ${ }^{1}$, Sri E.Venkata Narayana ${ }^{2}$ \\ ${ }^{1,2}$ Dept. Of Electronics and communication engineering, University College of Engineering, JNTUK, Kakinada, \\ East Godavari Dist, Andhra Pradesh, India.
}

\begin{abstract}
JPEG Compression is the most prevalent technique or method for images codecs. But it suffers from blocking artifacts. In this paper a comparison of the perceptual quality of deblocked images based on various quality assessments metric is done. A proposed PSNR including blocking effect factor was used instead of PSNR. Another quality assessment metric SSIM was used which produces results largely in accordance with PSNR-B. We show the simulation results, which prove PSNR-B produces objective judgments. The efficiency of deblocking algorithms was studied.
\end{abstract}

Keywords: Deblocked images, blocking artifacts, quality assessment, quality metric

\section{INTRODUCTION}

Many practical and commercial systems use digital image compression when it is required to transmit or store the image over limited resources. JPEG compression is the most popular image compression standard among all the members of lossy compression standards family. JPEG image coding is based on block based discrete cosine transform. BDCT coding has been successfully used in image and video compression applications due to its energy compacting property and relative ease of implementation. After segmenting an image in to blocks of size $\mathrm{N} \times \mathrm{N}$, the blocks are independently DCT transformed, quantized, coded and transmitted. One of the most noticeable degradation of the block transform coding is the "blocking artifact". These artifacts appear as a regular pattern of visible block boundaries. This degradation is the result of course quantization of the coefficients and of the independent processing of the blocks which does not take in to account the existing correlations among adjacent block pixels [12]. In order to achieve high compression rates using BTC with visually acceptable results, a procedure known as deblocking is done in order to eliminate blocking artifacts.

In this paper a research has done on quality assessment of deblocked images by estimating various quality metrics and the effect of quantization step of the measured quality of deblocked image is studied. Simulations are done using quality metrics such as peak signal-to-noise ratio (PSNR), structural similarity index (SSIM) and PSNR-B. PSNR-B is a new quality metric which includes PSNR by a blocking factor. By going through simulation results, it is shown that PSNR-B correlates well with the SSIM index and subjective quality and its performance is much better than the PSNR.

\section{QUALITY ASSESSMENT \& DEBLOCKING}

To remove blocking effect, several deblocking techniques have been proposed in the literature as post process mechanisms after JPEG compression, depending on the angle from which the blocking problem is tackled. If deblocking is viewed as an estimation problem, the simplest solution is probably just to low pass the blocky JPEG compressed image. More sophisticated methods involve iterative methods such as projection on convex sets $[3,4]$ and constrained least squares $[4,5]$ In this paper we use deblocking algorithms including lowpass filtering and projection on to convex sets. The efficiency of these algorithms can be analyzed by introducing a proposed method in the following section.

In this project We consider the two reference models class of quality assessment (QA) methods that are full-reference (FR) QA, which compares the test (distorted) image with a reference (original) image., the distorted images will ostensibly suffer from blocking artifacts or from the residual artifacts.

\section{PROPOSED METHOD}

Deblocking operation is performed in order to reduce blocking artifacts. Deblocking operation can be achieved by using various deblocking algorithms, employing deblocking filters. The effects of deblocking filters can be analyzed by introducing a change in distortion concept.

The deblocking operation results in the enhancement of image quality in some areas, while degrading in other areas. 


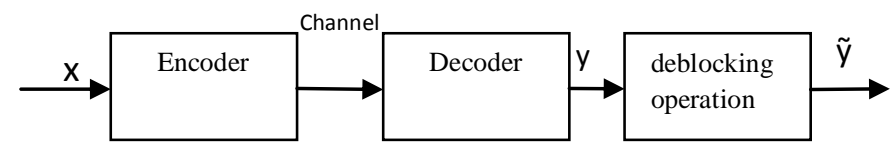

Fig 1 Block diagram showing JPEG compression

Let $\mathrm{X}$ be the reference image and $\mathrm{Y}$ be the test image (decoded image) distorted by quantization errors and $\tilde{Y}$ be the deblocked image as shown in figurel. Let $f$ represent the

Deblocking operation and is given by $\tilde{Y}=\mathrm{f}(\mathrm{Y})$. Let the quality metric between $\mathrm{X}$ and $\mathrm{Y}$ be $\mathrm{M}(\mathrm{X}, \mathrm{Y})$. For the given image $\mathrm{Y}$, the main aim of deblocking operation $\mathrm{f}$ is to maximize $\mathrm{M}(\mathrm{X}, \mathrm{f}(\mathrm{Y}))$.

\section{ESTIMATION OF QUALITY METRICS}

To Measure the quality degradation of an available distorted image with reference to the original image, a class of quality assessment metrics called full reference (FR) are considered. Full reference metrics perform distortion measures having full access to the original image. The quality assessment metrics are estimated as follows

\section{A. Peak signal to noise ratio}

The simplest and most widely used FR QA metrics are the peak signal-to-noise ratio (PSNR) and the mean-squared error (MSE) [1], [3].

It is most easily defined via the mean squared error (MSE) which for two $\mathrm{m} \times \mathrm{n}$ monochrome images I and $\mathrm{K}$ where one of the images is considered a noisy approximation of the other is defined as

Let $\mathrm{x}$ and $\mathrm{y}$ represent the vectors of reference and test image signals, respectively. Let $\mathrm{e}$ be the vector of error signal between $\mathrm{x}$ and $\mathrm{y}$. If the number of pixels in an image is $\mathrm{N}$

The PSNR is defined as:

$$
\operatorname{MSE}(x, y)=\frac{1}{N} \sum_{i=1}^{N} e_{i}^{2}=\frac{1}{N} \sum_{i=1}^{N}\left(x_{i}-y_{i}\right)^{2}
$$

$$
\operatorname{PSNR}(x, y)=\operatorname{10log}_{10} \frac{255^{2}}{\operatorname{MSE}(x, y)}
$$

\section{B. Structural similarity index metrics}

A product of three aspects of similarity is measured: luminance, contrast, and structure. The structural similarity (SSIM) metric aims to measure quality by capturing the similarity of images. The luminance comparison function $\mathrm{L}(\mathrm{x}, \mathrm{y})$ for reference image $\mathrm{x}$ and test image $\mathrm{y}$ is defined as

$$
I(x, y)=\frac{2 \mu_{x} \mu_{y}+C_{1}}{\mu_{x}^{2}+\mu_{y}^{2}+C_{1}}
$$

Where $\mu_{x}$ and $\mu_{y}$ are the mean values of $\mathrm{x}$ and $\mathrm{y}$, respectively, and $\mathrm{C} 1$ is a stabilizing constant. The contrast comparison function $\mathrm{C}(\mathrm{x}, \mathrm{y})$ is defined similarly as

$$
C(x, y)=\frac{2 \sigma_{x} \sigma_{y}+C_{2}}{\sigma_{x}^{2}+\sigma_{y}^{2}+C_{2}}
$$

Where $\sigma_{x}$ and $\sigma_{y}$ are the standard deviation of $\mathrm{x}$ and $\mathrm{y}$, respectively, and $\mathrm{C} 2$ is a stabilizing constant.

The structure comparison functions $\mathrm{S}(\mathrm{x}, \mathrm{y})$ is defined as

$$
S(x, y)=\frac{\sigma_{x y}+C_{3}}{\sigma_{x} \sigma_{y}+C_{3}}
$$

Where $\sigma_{x y}$ is the correlation between $\mathrm{x}$ and y and $\mathrm{C} 3$ is also a constant that provides stability.

The SSIM index is obtained by combining the three comparison functions

$$
\operatorname{SSIM}(x, y)=[\mathbf{l}(\mathbf{x}, \mathbf{y})]^{\alpha} \cdot[\mathbf{C}(\mathbf{x}, \mathbf{y})]^{\beta} \cdot[\mathbf{S}(\mathbf{x}, \mathbf{y})]^{\gamma}
$$

The parameters are set as

$$
\begin{gathered}
\alpha=\beta=\gamma=1 \text { And } C 3=C 2 / 2 \\
\operatorname{SSIM}(x, y)=\frac{\left(2 \mu_{x} \mu_{y}+C_{1}\right)\left(2 \sigma_{x y}+C_{2}\right)}{\left(\mu_{x}^{2}+\mu_{y}^{2}+C_{1}\right)\left(\mu_{x}^{2}+\mu_{y}^{2}+C_{2}\right)}
\end{gathered}
$$

Local SSIM statistics are estimated using a symmetric Gaussian weighting function. The mean SSIM index pools the spatial SSIM values to evaluate the overall image quality.

$$
\operatorname{SSIM}(x, y)=\frac{1}{M} \sum_{j=1}^{M} \operatorname{SSIM}\left(x_{j} \cdot y_{j}\right)
$$

Where $\mathrm{M}$ is the number of local windows over the image, and $x_{j}$ and $y_{j}$ are image patches covered by the jth window. 


\section{EFFECT OF QUANTIZATION STEP SIZE.}

The amount of compression and the quality can be controlled by the quantization step. Quantization is a key element of lossy compression, but information is lost. There are tradeoffs between compression ratio and reconstructed image/video quality. As the quantization step is increased, the compression ratio becomes larger, and the quality generally worsens. However, there has not been a study made of how perceptual quality suffers as a function of step size or the degree to which deblocking augments perceptual quality. The emergence of new and powerful IQA indices suggests this possibility.

In block transform coding, the input image is divided into LxL blocks, and each block is transformed independently into transform coefficients. An input image block is transformed into a DCT coefficient block

$$
\mathbf{B}=\mathbf{T B T}^{\mathbf{t}} \quad(\mathbf{9})
$$

Where $\mathrm{T}$ is the transform matrix and $T^{t}$ is the transpose matrix of $\mathrm{T}$. The transform coefficients are quantized using a scalar quantizer $\mathrm{Q}$

$$
\widetilde{B}=Q(B)=Q\left(\boldsymbol{T b T}^{t}\right)
$$

The quantization operator in (10) is nonlinear, and is a many-to-one mapping from $\mathbf{R}^{\mathbf{L}^{2}}$ to $\mathbf{R}^{\mathbf{L}^{2}}$.In the decoder, only quantized transform coefficients $\widetilde{B}$ are available.

The output of the decoder is

$$
\tilde{\mathbf{b}}=\mathbf{T}^{\mathrm{t}} \widetilde{\mathbf{B}} \mathbf{T}=\mathbf{T}^{\mathbf{t}} \mathbf{Q}\left(\mathbf{T b T}^{\mathrm{t}}\right) \mathbf{T}
$$

Let $\Delta$ represent the quantization step. It is well known that the PSNR is a monotonically decreasing function of $\Delta$. The SSIM index captures the similarity of reference and test images. As the quantization step size becomes larger, the structural differences between reference and test image will generally increase, and in particular the structure term $\mathrm{S}(\mathrm{X}, \mathrm{f})$ in (5) will become smaller. Hence, the SSIM index would be a monotonically decreasing function of the quantization step size.

\section{DEBLOCKING FILTER AND DISTORTION CHANGE}

As before, $\mathrm{x}$ is the reference (original) image and $\mathrm{y}$ is the decoded image that has been distorted by quantization errors. Let $\tilde{y}$ represent the de blocked image and f represent the deblocking operation: $\tilde{\mathbf{y}}=\mathbf{f}(\mathbf{y})$. Fig. 1 shows a block diagram depicting the flow of reference, decoded, and deblocked images.

Let $\mathbf{M}(\mathbf{x}, \mathbf{y})$ be the quality metric between and. The goal of the deblocking operation y is to maximize $\mathbf{M}(\mathbf{x}, \mathbf{f}(\mathbf{y}))$, given image. Deblocking is a local operation. The de blocking operation may improve the appearance of the image in some regions, while degrading the quality elsewhere.

Let $\mathbf{d}\left(\mathbf{x}_{\mathbf{i}}, \mathbf{y}_{\mathbf{i}}\right)$ be the distortion between the ith pixels of and, expressed as squared Euclidean distance

$$
\mathbf{d}\left(\mathbf{x}_{\mathbf{i}}, \mathbf{y}_{\mathbf{i}}\right)=\left\|\mathbf{x}_{\mathbf{i}}-\mathbf{y}_{\mathbf{i}}\right\|^{2}
$$

Next, we define the distortion decrease region (DDR) to be composed of those pixels where the distortion is decreased by the deblocking operation

$$
\mathbf{i} \in \mathbf{A}, \text { if } \mathbf{d}\left(\mathbf{x}_{\mathbf{i}}, \tilde{\mathbf{y}}_{\mathbf{i}}\right)<\boldsymbol{d}\left(\mathbf{x}_{\mathbf{i}}, \mathbf{y}_{\mathbf{i}}\right)
$$

The amount of distortion decrease for the ith pixel $\alpha_{i}$ in the DDRA is

$$
\alpha_{i}=d\left(x_{i} \cdot y_{i}\right)-d\left(x_{i}, \tilde{y}_{i}\right)
$$

The distortion may also increase at other pixels by application of the deblocking filter. We similarly define the distortion increase region (DIR) B

$$
\mathbf{i} \in \mathbf{B}, \text { if } \mathbf{d}\left(\mathbf{x}_{\mathbf{i}}, \mathbf{y}_{\mathrm{i}}\right)<\boldsymbol{d}\left(\mathrm{x}_{\mathbf{i}}, \tilde{\mathbf{y}}_{\mathrm{i}}\right)
$$

The amount of distortion increase for the ith pixel $\beta_{i}$ in the DIRB is

We define the mean distortion decrease (MDD)

$$
\beta_{\mathrm{i}}=\mathbf{d}\left(\mathbf{x}_{\mathrm{i}}, \tilde{\mathbf{y}}_{\mathbf{i}}\right)-\mathbf{d}\left(\mathbf{x}_{\mathrm{i}}, \mathbf{y}_{\mathbf{i}}\right)
$$

$$
\overline{\boldsymbol{\alpha}}=\frac{1}{\mathbf{N}} \sum_{\mathrm{i} \in \mathrm{A}}\left(\mathrm{d}\left(\mathrm{x}_{\mathrm{i}}, \mathbf{y}_{\mathrm{i}}\right)-\mathbf{d}\left(\mathrm{x}_{\mathrm{i}}, \tilde{\mathbf{y}}_{\mathrm{i}}\right)\right.
$$

Where $\mathrm{N}$ is the number of pixels in the image. Similarly the mean distortion increase (MDI) is

$$
\overline{\boldsymbol{\beta}}=\frac{1}{\mathbf{N}} \sum_{\mathbf{i} \in \mathbf{B}}\left(\mathbf{d}\left(\mathbf{x}_{\mathbf{i}}, \tilde{\mathbf{y}}_{\mathbf{i}}\right)-\mathbf{d}\left(\mathbf{x}_{\mathbf{i}}, \mathbf{y}_{\mathbf{i}}\right)\right.
$$

A reasonable approach for designing a deblocking filter would be to seek to maximize the MDD $\bar{\alpha}$ and minimize the MDI $\bar{\beta}$. This is generally a very difficult task and of course, may not result in optimized improvement in perceptual quality. Lastly, let be the mean distortion change (MDC), defined as the difference between MDD and MDI

$$
\overline{\boldsymbol{\gamma}}=\overline{\boldsymbol{\alpha}}-\overline{\boldsymbol{\beta}}
$$

If $\bar{\gamma}<0$, then the deblocking operation is likely unsuccessful since the mean distortion increase is larger than the mean distortion decrease. We would expect a successful deblocking operation to yield $\bar{\gamma}>0$. Nevertheless, these conditions are not equated with levels of perceptual improvement or loss. Deblocking can be considered as an image restoration problem. Let represent the deblocking operation function and $N\left(x_{i}\right)$ represent a 
neighbourhood of pixel $x_{i}$. A lowpass filter is a simple deblocking filter. An LxL low pass filter can be represented as

$$
\mathbf{g}\left(\mathrm{N}\left(\mathbf{x}_{\mathbf{i}}\right)\right)=\sum_{\mathrm{k}=1}^{\mathrm{L}^{2}} \mathbf{h}_{\mathbf{k}} \cdot \mathbf{x}_{\mathbf{i}, \mathrm{K}} \ldots \ldots
$$

Where $h_{k}$ is the kernel for the LxL filter and is the th pixel in the neighbourhood of pixel $x_{i}$. While lowpass filtering does reduce blocking artifacts, critical high frequency information is also lost and the image is blurred. While the distortion will certainly decrease for some pixels that define the DDR, the distortion will likely increase for a significant number of pixels in DIR. Indeed, it is quite possible that $\bar{\gamma}<0$ could result. Moreover, blur is perceptually annoying. A variety of nonlinear methods have been proposed to reduce the blocking artifacts, while minimizing the loss of original information. For example, deblocking algorithms based upon projection onto convex sets (POCS) have demonstrated good performance for reducing blocking artifacts and have proved popular.

In POCS, a low pass filtering operation is performed in the spatial domain, while a projection operation is performed in the DCT domain. Typically, the projection operation is a clipping operation on the filtered coefficients, confining these to fall within a certain range defined by the quantization step size. Since the low pass filtering and the projection operations are performed in different domains, forward DCT and inverse DCT (IDCT) operations are required. The low pass filtering, DCT, projection, IDCT operations compose one iteration, and multiple iterations are required to achieve convergence. It is argued that under certain conditions, POCS filtered images converge to an image that does not exhibit blocking artifacts.

\section{PSNR INCLUDING BLOCKING EFFECTS}

In the following, we propose a new block-sensitive image quality metric which we term peak signal-tonoise ratio including blocking effects (PSNR-B). As the quantization step size increases, blocking artifacts generally become more conspicuous. Blocking artifacts are gray level discontinuities at block boundaries, which are ordinarily oriented horizontally and vertically. They arise from poor representation of the block luminance levels near the block boundaries. The following definitions are relative to an assumed block-based compression tiling, e.g., 8x8 blocks as in JPEG compression. For simplicity, assume that an integer number of blocks comprise the image, viz., that horizontal and vertical dimensions are divisible by the block dimension. The definitions apply whether the image is compressed, not-compressed, or deblocked following decompression.

We, therefore, consider blocking artifacts that occur along the horizontal and vertical orientations. Let $\boldsymbol{N}_{\boldsymbol{H}}$ and $\boldsymbol{N}_{\boldsymbol{v}}$ be the horizontal and vertical dimensions of the $\boldsymbol{N}_{\boldsymbol{H}} \boldsymbol{X} \boldsymbol{N}_{\boldsymbol{v}}$ image I. Let $\mathcal{H}$ be the set of horizontal neighbouring pixel pairs in I. Let $\mathcal{H}_{\boldsymbol{B}} \subset \mathcal{H}$ be the set of horizontal neighbouring pixel pairs that lie across a

\begin{tabular}{|c|c|c|c|c|c|c|c|}
\hline $\mathbf{y}_{1}$ & $\mathbf{y}_{2}$ & $\mathbf{y}_{\mathbf{3}}$ & $\mathbf{y}_{4}$ & $\mathbf{y}_{5}$ & $\mathbf{y}_{6}$ & $\mathbf{y}_{7}$ & $\mathbf{y}_{8}$ \\
\hline $\mathbf{y}_{9}$ & $\mathbf{y}_{10}$ & $\mathbf{y}_{11}$ & $\mathbf{y}_{12}$ & $\mathbf{y}_{13}$ & $\mathbf{y}_{14}$ & $\mathbf{y}_{15}$ & $\mathbf{y}_{16}$ \\
\hline $\mathbf{y}_{17}$ & $\mathbf{y}_{18}$ & $\mathbf{y}_{19}$ & $\mathbf{y}_{20}$ & $\mathbf{y}_{21}$ & $\mathbf{y}_{22}$ & $\mathbf{y}_{23}$ & $\mathbf{y}_{24}$ \\
\hline $\mathbf{y}_{25}$ & $\mathbf{y}_{26}$ & $\mathbf{y}_{27}$ & $\mathbf{y}_{28}$ & $\mathbf{y}_{29}$ & $\mathbf{y}_{30}$ & $\mathbf{y}_{31}$ & $\mathbf{y}_{32}$ \\
\hline $\mathbf{y}_{33}$ & $\mathbf{y}_{34}$ & $\mathbf{y}_{35}$ & $\mathbf{y}_{36}$ & $\mathbf{y}_{37}$ & $\mathbf{y}_{\mathbf{3 8}}$ & $\mathbf{y}_{39}$ & $\mathbf{y}_{40}$ \\
\hline $\mathbf{y}_{41}$ & $\mathbf{y}_{42}$ & $\mathbf{y}_{43}$ & $\mathbf{y}_{44}$ & $\mathbf{y}_{45}$ & $\mathbf{y}_{46}$ & $\mathbf{y}_{47}$ & $\mathbf{y}_{48}$ \\
\hline$y_{49}$ & $\mathbf{y}_{50}$ & $\mathbf{y}_{51}$ & $\mathbf{y}_{52}$ & $\mathbf{y}_{53}$ & $\mathbf{y}_{54}$ & $\mathbf{y}_{55}$ & $\mathbf{y}_{56}$ \\
\hline $\mathbf{y}_{57}$ & $\mathbf{y}_{58}$ & $\mathbf{y}_{59}$ & $\mathbf{y}_{60}$ & $\mathbf{y}_{61}$ & $\mathbf{y}_{62}$ & $\mathbf{y}_{63}$ & $\mathbf{y}_{64}$ \\
\hline
\end{tabular}

block boundary. Let $\boldsymbol{H}_{B}^{\mathcal{C}}$ be the set of horizontal neighbouring pixel pairs, not lying across a block boundary,

i.e. $\mathcal{H}_{B}^{C}=\mathcal{H}-\mathcal{H}_{B}$, . Similarly, let $v$ be the set of vertical neighbouring pixel pairs, and $\boldsymbol{v}_{\boldsymbol{B}}$ be the set of vertical neighbouring pixel pairs lying across block boundaries. Let $\boldsymbol{v}_{\boldsymbol{B}}^{\boldsymbol{C}}$ be the set of vertical neighbouring pixel pairs not lying across block boundaries i.e. $\boldsymbol{v}_{B}^{C}=\boldsymbol{v}-\boldsymbol{v}_{B}$.

Let $\boldsymbol{N}_{\boldsymbol{H}_{B}}, \boldsymbol{N}_{\boldsymbol{H}_{B}^{C}}, \boldsymbol{N}_{\boldsymbol{V}_{B}}, \boldsymbol{N}_{V_{B}^{c}}$ be the number of pixel pairs in $\mathcal{H}_{B}, \mathcal{H}_{B}^{C}, \boldsymbol{v}_{\boldsymbol{B}}$ and $\boldsymbol{v}_{\boldsymbol{B}}^{\boldsymbol{C}}$ respectively .If B is the block size then

$$
\begin{aligned}
& \mathbf{N H}_{B}=\mathbf{N}_{\mathbf{V}}\left(\frac{\mathbf{N}_{H}}{B}-\mathbf{1}\right) \\
& \mathbf{N}_{\mathbf{H}_{B}^{C}}=\mathbf{N}_{\mathbf{V}}\left(\mathbf{N}_{\mathbf{H}}-\mathbf{1}\right)-\mathbf{N}_{\mathbf{H}_{B}} \\
& \mathbf{N}_{\mathbf{V}_{B}}=\mathbf{N}_{\mathbf{H}}\left(\frac{\mathbf{N}_{\mathbf{V}}}{B}-\mathbf{1}\right) \\
& \mathbf{N}_{\mathbf{V}_{B}^{C}}=\mathbf{N}_{\mathbf{H}}\left(\mathbf{N}_{\mathbf{V}}-\mathbf{1}\right)-\mathbf{N}_{\mathbf{V}_{B}}
\end{aligned}
$$

Fig. 2(a) example for illustration of (8x8) pixel blocks 


\begin{tabular}{|c|c|c|c|c|c|c|c|c|c|c|c|c|c|c|c|}
\hline $\begin{array}{l}\mathbf{y} \\
1\end{array}$ & $\begin{array}{l}\mathbf{y} \\
\mathbf{2}\end{array}$ & $\begin{array}{l}\mathbf{y} \\
\mathbf{3}\end{array}$ & $\begin{array}{l}\mathbf{y} \\
4\end{array}$ & $\begin{array}{l}\mathbf{y} \\
5\end{array}$ & $\begin{array}{l}\mathbf{y} \\
6\end{array}$ & $\begin{array}{l}\mathbf{y} \\
7\end{array}$ & $\begin{array}{l}\mathbf{y} \\
8\end{array}$ & $\begin{array}{l}\mathbf{y} \\
\mathbf{9}\end{array}$ & $\begin{array}{l}\mathbf{y} \\
\mathbf{1} \\
\mathbf{0}\end{array}$ & $\begin{array}{l}\mathbf{y} \\
\mathbf{1} \\
\mathbf{1}\end{array}$ & $\begin{array}{l}\mathbf{y} \\
\mathbf{1} \\
\mathbf{2}\end{array}$ & $\begin{array}{l}\mathbf{y} \\
\mathbf{1} \\
\mathbf{3}\end{array}$ & $\begin{array}{l}\mathbf{y} \\
1 \\
4\end{array}$ & $\begin{array}{l}\mathbf{y} \\
\mathbf{1} \\
5\end{array}$ & $\begin{array}{l}y \\
1 \\
6\end{array}$ \\
\hline $\begin{array}{l}\mathbf{y} \\
1 \\
7\end{array}$ & $\begin{array}{l}\mathbf{y} \\
1 \\
8\end{array}$ & $\begin{array}{l}\mathbf{y} \\
1 \\
\mathbf{9}\end{array}$ & $\begin{array}{l}\mathbf{y} \\
\mathbf{2} \\
\mathbf{0}\end{array}$ & $\begin{array}{l}\mathbf{y} \\
2 \\
1\end{array}$ & $\begin{array}{l}\mathbf{y} \\
2 \\
2\end{array}$ & $\mathbf{y}$ & $\begin{array}{l}y \\
2 \\
4\end{array}$ & $\begin{array}{l}\mathbf{y} \\
\mathbf{2} \\
5\end{array}$ & $\begin{array}{l}y \\
2 \\
6\end{array}$ & $\begin{array}{l}\mathbf{y} \\
2 \\
7\end{array}$ & $\begin{array}{l}\mathbf{y} \\
2 \\
8\end{array}$ & $\begin{array}{l}\mathbf{y} \\
2 \\
9\end{array}$ & $\begin{array}{l}\mathbf{y} \\
\mathbf{3} \\
\mathbf{0}\end{array}$ & $\begin{array}{l}\mathbf{y} \\
\mathbf{3} \\
\mathbf{1}\end{array}$ & $\begin{array}{l}\mathbf{y} \\
\mathbf{3} \\
\mathbf{2}\end{array}$ \\
\hline $\begin{array}{l}\mathbf{y} \\
\mathbf{3} \\
\mathbf{3}\end{array}$ & $\begin{array}{l}\mathbf{y} \\
\mathbf{3} \\
\mathbf{4}\end{array}$ & $\begin{array}{l}\mathbf{y} \\
\mathbf{3} \\
\mathbf{5}\end{array}$ & $\begin{array}{l}\mathbf{y} \\
3 \\
6\end{array}$ & $\begin{array}{l}\mathbf{y} \\
\mathbf{3} \\
\mathbf{7}\end{array}$ & $\begin{array}{l}\mathbf{y} \\
\mathbf{3} \\
\mathbf{8}\end{array}$ & $\begin{array}{l}\mathbf{y} \\
\mathbf{3} \\
\mathbf{9}\end{array}$ & $\begin{array}{l}y \\
4 \\
0\end{array}$ & $\begin{array}{l}y \\
4 \\
1\end{array}$ & $\begin{array}{l}y \\
4 \\
2\end{array}$ & $\begin{array}{l}y \\
4 \\
3\end{array}$ & $\begin{array}{l}y \\
4 \\
4\end{array}$ & $\begin{array}{l}y \\
4 \\
5\end{array}$ & $\begin{array}{l}y \\
4 \\
6\end{array}$ & $\begin{array}{l}y \\
4 \\
7\end{array}$ & $\begin{array}{l}y \\
4 \\
8\end{array}$ \\
\hline $\begin{array}{l}y \\
4 \\
9\end{array}$ & $\begin{array}{l}\mathbf{y} \\
\mathbf{5} \\
\mathbf{0}\end{array}$ & $\begin{array}{l}\mathbf{y} \\
5 \\
1\end{array}$ & $\begin{array}{l}\mathbf{y} \\
5 \\
2\end{array}$ & $\begin{array}{l}\mathbf{y} \\
5 \\
3\end{array}$ & $\begin{array}{l}y \\
5 \\
4\end{array}$ & 5 & $\begin{array}{l}y \\
5 \\
6\end{array}$ & $\begin{array}{l}\mathbf{y} \\
5 \\
7\end{array}$ & $\begin{array}{l}\mathbf{y} \\
5 \\
8\end{array}$ & $\begin{array}{l}\mathbf{y} \\
\mathbf{5} \\
\mathbf{9}\end{array}$ & $\begin{array}{l}\mathbf{y} \\
6 \\
0\end{array}$ & $\begin{array}{l}y \\
6 \\
1\end{array}$ & $\begin{array}{l}\mathbf{y} \\
6 \\
2\end{array}$ & $\begin{array}{l}\mathbf{y} \\
6 \\
3\end{array}$ & $\begin{array}{l}y \\
6 \\
4\end{array}$ \\
\hline $\begin{array}{l}y \\
6 \\
5\end{array}$ & $\begin{array}{l}y \\
6 \\
6\end{array}$ & $\begin{array}{l}y \\
6 \\
7\end{array}$ & $\begin{array}{l}y \\
6 \\
8\end{array}$ & $\begin{array}{l}\mathbf{y} \\
6 \\
9\end{array}$ & $\begin{array}{l}\mathbf{y} \\
7 \\
\mathbf{0}\end{array}$ & 1 & $\begin{array}{l}\mathbf{y} \\
7 \\
2\end{array}$ & $\begin{array}{l}\mathbf{y} \\
7 \\
\mathbf{3}\end{array}$ & $\begin{array}{l}\mathbf{y} \\
7 \\
4\end{array}$ & $\begin{array}{l}\mathbf{y} \\
7 \\
5\end{array}$ & $\begin{array}{l}y \\
7 \\
6\end{array}$ & $\begin{array}{l}\mathbf{y} \\
7 \\
7\end{array}$ & $\begin{array}{l}\mathbf{y} \\
7 \\
8\end{array}$ & $\begin{array}{l}\mathbf{y} \\
7 \\
\mathbf{9}\end{array}$ & $\begin{array}{l}\mathbf{y} \\
\mathbf{8} \\
\mathbf{0}\end{array}$ \\
\hline $\begin{array}{l}\mathbf{y} \\
8 \\
1\end{array}$ & $\begin{array}{l}\mathbf{y} \\
8 \\
2\end{array}$ & $\begin{array}{l}\mathbf{y} \\
\mathbf{8} \\
\mathbf{3}\end{array}$ & $\begin{array}{l}\mathbf{y} \\
8 \\
4\end{array}$ & $\begin{array}{l}\mathbf{y} \\
8 \\
5\end{array}$ & $\begin{array}{l}y \\
8 \\
6\end{array}$ & $\begin{array}{l}\mathbf{y} \\
8\end{array}$ & $\begin{array}{l}\mathbf{y} \\
8 \\
8\end{array}$ & $\begin{array}{l}\mathbf{y} \\
8 \\
9\end{array}$ & $\begin{array}{l}\mathbf{y} \\
\mathbf{9} \\
\mathbf{0}\end{array}$ & $\begin{array}{l}\mathbf{y} \\
\mathbf{9} \\
\mathbf{1}\end{array}$ & $\begin{array}{l}\mathbf{y} \\
9 \\
2\end{array}$ & $\begin{array}{l}\mathbf{y} \\
\mathbf{9} \\
\mathbf{3}\end{array}$ & $\begin{array}{l}\mathbf{y} \\
\mathbf{9} \\
4\end{array}$ & $\begin{array}{l}\mathbf{y} \\
9 \\
5\end{array}$ & $\begin{array}{l}y \\
9 \\
6\end{array}$ \\
\hline $\begin{array}{l}\mathbf{y} \\
9 \\
7\end{array}$ & $\begin{array}{l}\mathbf{y} \\
9 \\
8\end{array}$ & $\begin{array}{l}\mathbf{y} \\
9 \\
9\end{array}$ & $\begin{array}{l}\mathbf{y} \\
\mathbf{1} \\
\mathbf{0} \\
\mathbf{0}\end{array}$ & $\begin{array}{l}\mathbf{y} \\
\mathbf{1} \\
\mathbf{0} \\
\mathbf{1}\end{array}$ & $\begin{array}{l}\mathbf{y} \\
\mathbf{1} \\
\mathbf{0} \\
\mathbf{2}\end{array}$ & 3 & $\begin{array}{l}\mathbf{y} \\
1 \\
0 \\
4\end{array}$ & $\begin{array}{l}\mathbf{y} \\
\mathbf{1} \\
\mathbf{0} \\
\mathbf{5}\end{array}$ & $\begin{array}{l}\mathbf{y} \\
1 \\
0 \\
6\end{array}$ & $\begin{array}{l}\mathbf{y} \\
\mathbf{1} \\
\mathbf{0} \\
7\end{array}$ & $\begin{array}{l}\mathbf{y} \\
\mathbf{1} \\
\mathbf{0} \\
\mathbf{8}\end{array}$ & $\begin{array}{l}\mathbf{y} \\
\mathbf{1} \\
\mathbf{0} \\
\mathbf{9}\end{array}$ & $\begin{array}{l}\mathbf{y} \\
\mathbf{1} \\
\mathbf{1} \\
\mathbf{0}\end{array}$ & $\begin{array}{l}\mathbf{y} \\
\mathbf{1} \\
\mathbf{1} \\
\mathbf{1}\end{array}$ & $\begin{array}{l}\mathbf{y} \\
\mathbf{1} \\
\mathbf{1} \\
\mathbf{2}\end{array}$ \\
\hline $\begin{array}{l}\mathbf{y} \\
\mathbf{1} \\
\mathbf{1} \\
\mathbf{3}\end{array}$ & $\begin{array}{l}\mathbf{y} \\
1 \\
1 \\
4\end{array}$ & $\begin{array}{l}\mathbf{y} \\
\mathbf{1} \\
\mathbf{1} \\
\mathbf{5}\end{array}$ & $\begin{array}{l}\mathbf{y} \\
\mathbf{1} \\
\mathbf{1} \\
\mathbf{6}\end{array}$ & $\begin{array}{l}\mathbf{y} \\
\mathbf{1} \\
\mathbf{1} \\
\mathbf{7}\end{array}$ & $\begin{array}{l}\mathbf{y} \\
\mathbf{1} \\
\mathbf{1} \\
\mathbf{8}\end{array}$ & $\begin{array}{l}\mathbf{y} \\
\mathbf{1}\end{array}$ & $\begin{array}{l}\mathbf{y} \\
\mathbf{1} \\
\mathbf{2} \\
\mathbf{0}\end{array}$ & $\begin{array}{l}\mathbf{y} \\
\mathbf{1} \\
\mathbf{2} \\
\mathbf{1}\end{array}$ & $\begin{array}{l}\mathbf{y} \\
\mathbf{1} \\
\mathbf{2} \\
\mathbf{2}\end{array}$ & $\begin{array}{l}\mathbf{y} \\
\mathbf{1} \\
\mathbf{2} \\
\mathbf{3}\end{array}$ & $\begin{array}{l}\mathbf{y} \\
\mathbf{1} \\
\mathbf{2} \\
\mathbf{4}\end{array}$ & $\begin{array}{l}\mathbf{y} \\
\mathbf{1} \\
\mathbf{2} \\
\mathbf{5}\end{array}$ & $\begin{array}{l}\mathbf{y} \\
\mathbf{1} \\
\mathbf{2} \\
\mathbf{6}\end{array}$ & $\begin{array}{l}\mathbf{y} \\
1 \\
2 \\
7\end{array}$ & $\begin{array}{l}\mathbf{y} \\
1 \\
2 \\
8\end{array}$ \\
\hline $\begin{array}{l}\mathbf{y} \\
1 \\
2 \\
9\end{array}$ & $\begin{array}{l}\mathbf{y} \\
\mathbf{1} \\
\mathbf{3} \\
\mathbf{0}\end{array}$ & $\begin{array}{l}\mathbf{y} \\
\mathbf{1} \\
\mathbf{3} \\
\mathbf{1}\end{array}$ & $\begin{array}{l}\mathbf{y} \\
\mathbf{1} \\
\mathbf{3} \\
\mathbf{2}\end{array}$ & $\begin{array}{l}\mathbf{y} \\
\mathbf{1} \\
\mathbf{3} \\
\mathbf{3}\end{array}$ & $\begin{array}{l}\mathbf{y} \\
\mathbf{1} \\
\mathbf{3} \\
\mathbf{4}\end{array}$ & 5 & $\begin{array}{l}\mathbf{y} \\
\mathbf{1} \\
\mathbf{3} \\
\mathbf{6}\end{array}$ & $\begin{array}{l}\mathbf{y} \\
\mathbf{1} \\
\mathbf{3} \\
\mathbf{7}\end{array}$ & $\begin{array}{l}\mathbf{y} \\
\mathbf{1} \\
\mathbf{3} \\
\mathbf{8}\end{array}$ & $\begin{array}{l}\mathbf{y} \\
\mathbf{1} \\
\mathbf{3} \\
\mathbf{9}\end{array}$ & $\begin{array}{l}y \\
1 \\
4 \\
0\end{array}$ & $\begin{array}{l}y \\
1 \\
4 \\
1\end{array}$ & $\begin{array}{l}\mathbf{y} \\
\mathbf{1} \\
\mathbf{4} \\
\mathbf{2}\end{array}$ & $\begin{array}{l}y \\
1 \\
4 \\
3\end{array}$ & $\begin{array}{l}y \\
1 \\
4 \\
4\end{array}$ \\
\hline $\begin{array}{l}y \\
1 \\
4 \\
5\end{array}$ & $\begin{array}{l}y \\
1 \\
4 \\
6\end{array}$ & $\begin{array}{l}y \\
1 \\
4 \\
7\end{array}$ & $\begin{array}{l}y \\
1 \\
4 \\
8\end{array}$ & $\begin{array}{l}\mathbf{y} \\
1 \\
4 \\
9\end{array}$ & $\begin{array}{l}\mathbf{y} \\
\mathbf{1} \\
\mathbf{5} \\
\mathbf{0}\end{array}$ & $\begin{array}{l}\mathbf{y} \\
\mathbf{1} \\
5\end{array}$ & $\begin{array}{l}\mathbf{y} \\
\mathbf{1} \\
\mathbf{5} \\
\mathbf{2}\end{array}$ & $\begin{array}{l}\mathbf{y} \\
\mathbf{1} \\
\mathbf{5} \\
\mathbf{3}\end{array}$ & $\begin{array}{l}\mathbf{y} \\
\mathbf{1} \\
\mathbf{5} \\
\mathbf{4}\end{array}$ & $\begin{array}{l}\mathbf{y} \\
1 \\
5 \\
5\end{array}$ & $\begin{array}{l}\mathbf{y} \\
\mathbf{1} \\
\mathbf{5} \\
\mathbf{6}\end{array}$ & $\begin{array}{l}\mathbf{y} \\
\mathbf{1} \\
5 \\
7\end{array}$ & $\begin{array}{l}\mathbf{y} \\
\mathbf{1} \\
\mathbf{5} \\
\mathbf{8}\end{array}$ & $\begin{array}{l}\mathbf{y} \\
1 \\
5 \\
9\end{array}$ & $\begin{array}{l}y \\
1 \\
6 \\
0\end{array}$ \\
\hline $\begin{array}{l}y \\
1 \\
6 \\
1\end{array}$ & $\begin{array}{l}y \\
1 \\
6 \\
2\end{array}$ & $\begin{array}{l}\mathbf{y} \\
1 \\
6 \\
3\end{array}$ & $\begin{array}{l}y \\
1 \\
6 \\
4\end{array}$ & $\begin{array}{l}y \\
1 \\
6 \\
5\end{array}$ & $\begin{array}{l}y \\
1 \\
6 \\
6\end{array}$ & $\begin{array}{l}\mathbf{y} \\
1\end{array}$ & $\begin{array}{l}\mathbf{y} \\
1 \\
6 \\
8\end{array}$ & $\begin{array}{l}y \\
1 \\
6 \\
9\end{array}$ & $\begin{array}{l}\mathbf{y} \\
\mathbf{1} \\
\mathbf{7} \\
\mathbf{0}\end{array}$ & $\begin{array}{l}\mathbf{y} \\
1 \\
7 \\
1\end{array}$ & $\begin{array}{l}\mathbf{y} \\
1 \\
7 \\
2\end{array}$ & $\begin{array}{l}\mathbf{y} \\
\mathbf{1} \\
\mathbf{7} \\
\mathbf{3}\end{array}$ & $\begin{array}{l}\mathbf{y} \\
\mathbf{1} \\
\mathbf{7} \\
\mathbf{4}\end{array}$ & $\begin{array}{l}\mathbf{y} \\
\mathbf{1} \\
7 \\
5\end{array}$ & $\begin{array}{l}\mathbf{y} \\
1 \\
7 \\
6\end{array}$ \\
\hline $\begin{array}{l}\mathbf{y} \\
1 \\
7 \\
7\end{array}$ & $\begin{array}{l}\mathbf{y} \\
\mathbf{1} \\
7 \\
8\end{array}$ & $\begin{array}{l}\mathbf{y} \\
\mathbf{1} \\
\mathbf{7} \\
\mathbf{9}\end{array}$ & $\begin{array}{l}\mathbf{y} \\
\mathbf{1} \\
\mathbf{8} \\
\mathbf{0}\end{array}$ & $\begin{array}{l}\mathbf{y} \\
\mathbf{1} \\
\mathbf{8} \\
\mathbf{1}\end{array}$ & $\begin{array}{l}\mathbf{y} \\
\mathbf{1} \\
\mathbf{8} \\
\mathbf{2}\end{array}$ & $\begin{array}{l}\mathbf{y} \\
\mathbf{1}\end{array}$ & $\begin{array}{l}\mathbf{y} \\
1 \\
8 \\
4\end{array}$ & $\begin{array}{l}\mathbf{y} \\
\mathbf{1} \\
\mathbf{8} \\
\mathbf{5}\end{array}$ & $\begin{array}{l}\mathbf{y} \\
1 \\
8 \\
6\end{array}$ & $\begin{array}{l}\mathbf{y} \\
\mathbf{1} \\
\mathbf{8} \\
7\end{array}$ & $\begin{array}{l}\mathbf{y} \\
1 \\
8 \\
8\end{array}$ & $\begin{array}{l}\mathbf{y} \\
1 \\
8 \\
9\end{array}$ & $\begin{array}{l}\mathbf{y} \\
\mathbf{1} \\
\mathbf{9} \\
\mathbf{0}\end{array}$ & $\begin{array}{l}\mathbf{y} \\
\mathbf{1} \\
\mathbf{9} \\
\mathbf{1}\end{array}$ & $\begin{array}{l}\mathbf{y} \\
1 \\
9 \\
2\end{array}$ \\
\hline $\begin{array}{l}\mathbf{y} \\
\mathbf{1} \\
\mathbf{9} \\
\mathbf{3}\end{array}$ & $\begin{array}{l}y \\
1 \\
9 \\
4\end{array}$ & $\begin{array}{l}\mathbf{y} \\
1 \\
9 \\
5\end{array}$ & $\begin{array}{l}\mathbf{y} \\
1 \\
9 \\
6\end{array}$ & $\begin{array}{l}\mathbf{y} \\
1 \\
\mathbf{9} \\
7\end{array}$ & $\begin{array}{l}\mathbf{y} \\
1 \\
9 \\
8\end{array}$ & $\begin{array}{l}\mathbf{y} \\
1\end{array}$ & $\begin{array}{l}\mathbf{y} \\
\mathbf{2} \\
\mathbf{0} \\
\mathbf{0}\end{array}$ & $\begin{array}{l}\mathbf{y} \\
\mathbf{2} \\
\mathbf{0} \\
\mathbf{1}\end{array}$ & $\begin{array}{l}\mathbf{y} \\
\mathbf{2} \\
\mathbf{0} \\
\mathbf{2}\end{array}$ & $\begin{array}{l}\mathbf{y} \\
\mathbf{2} \\
\mathbf{0} \\
\mathbf{3}\end{array}$ & $\begin{array}{l}\mathbf{y} \\
2 \\
0 \\
4\end{array}$ & $\begin{array}{l}\mathbf{y} \\
\mathbf{2} \\
\mathbf{0} \\
\mathbf{5}\end{array}$ & $\begin{array}{l}\mathbf{y} \\
2 \\
0 \\
6\end{array}$ & $\begin{array}{l}\mathbf{y} \\
\mathbf{2} \\
\mathbf{0} \\
7\end{array}$ & $\begin{array}{l}\mathbf{y} \\
2 \\
0 \\
8\end{array}$ \\
\hline $\begin{array}{l}\mathbf{y} \\
\mathbf{2} \\
\mathbf{0} \\
\mathbf{9}\end{array}$ & $\begin{array}{l}\mathbf{y} \\
\mathbf{2} \\
\mathbf{1} \\
\mathbf{0}\end{array}$ & $\begin{array}{l}\mathbf{y} \\
\mathbf{2} \\
\mathbf{1} \\
\mathbf{1}\end{array}$ & $\begin{array}{l}\mathbf{y} \\
2 \\
1 \\
2\end{array}$ & $\begin{array}{l}\mathbf{y} \\
\mathbf{2} \\
\mathbf{1} \\
\mathbf{3}\end{array}$ & $\begin{array}{l}\mathbf{y} \\
\mathbf{2} \\
1\end{array}$ & $\begin{array}{l}\mathbf{y} \\
\mathbf{2}\end{array}$ & $\begin{array}{l}\mathbf{y} \\
\mathbf{2} \\
\mathbf{1} \\
\mathbf{6}\end{array}$ & $\begin{array}{l}\mathbf{y} \\
\mathbf{2} \\
\mathbf{1} \\
\mathbf{7}\end{array}$ & $\begin{array}{l}\mathbf{y} \\
\mathbf{2} \\
\mathbf{1}\end{array}$ & $\begin{array}{l}\mathbf{y} \\
\mathbf{2} \\
\mathbf{1} \\
\mathbf{9}\end{array}$ & $\begin{array}{l}\mathbf{y} \\
\mathbf{2} \\
\mathbf{2} \\
\mathbf{0}\end{array}$ & $\begin{array}{l}y \\
2 \\
2 \\
1\end{array}$ & $\begin{array}{l}\mathbf{y} \\
\mathbf{2} \\
\mathbf{2} \\
\mathbf{2}\end{array}$ & $\begin{array}{l}\mathbf{y} \\
\mathbf{2} \\
\mathbf{2} \\
\mathbf{3}\end{array}$ & $\begin{array}{l}\mathbf{y} \\
2 \\
2 \\
4\end{array}$ \\
\hline $\begin{array}{l}\mathbf{y} \\
2 \\
2 \\
5\end{array}$ & $\begin{array}{l}\mathbf{y} \\
2 \\
2 \\
6\end{array}$ & $\begin{array}{l}\mathbf{y} \\
2 \\
2 \\
7\end{array}$ & $\begin{array}{l}\mathbf{y} \\
2 \\
2 \\
8\end{array}$ & $\begin{array}{l}\mathbf{y} \\
\mathbf{2} \\
\mathbf{2} \\
\mathbf{9}\end{array}$ & $\begin{array}{l}\mathbf{y} \\
\mathbf{2} \\
\mathbf{3} \\
\mathbf{0}\end{array}$ & $\begin{array}{l}\mathbf{y} \\
\mathbf{2} \\
\mathbf{3} \\
\mathbf{1}\end{array}$ & $\begin{array}{l}\mathbf{y} \\
\mathbf{2} \\
\mathbf{3} \\
\mathbf{2}\end{array}$ & $\begin{array}{l}\mathbf{y} \\
\mathbf{2} \\
\mathbf{3} \\
\mathbf{3}\end{array}$ & $\begin{array}{l}\mathbf{y} \\
2 \\
3 \\
4\end{array}$ & $\begin{array}{l}\mathbf{y} \\
2 \\
3 \\
5\end{array}$ & $\begin{array}{l}\mathbf{y} \\
2 \\
3 \\
6\end{array}$ & $\begin{array}{l}\mathbf{y} \\
\mathbf{2} \\
\mathbf{3} \\
7\end{array}$ & $\begin{array}{l}\mathbf{y} \\
\mathbf{2} \\
\mathbf{3} \\
\mathbf{8}\end{array}$ & $\begin{array}{l}\mathbf{y} \\
\mathbf{2} \\
\mathbf{3} \\
\mathbf{9}\end{array}$ & $\begin{array}{l}\mathbf{y} \\
2 \\
4 \\
0\end{array}$ \\
\hline $\begin{array}{l}y \\
2 \\
4 \\
1\end{array}$ & $\begin{array}{l}y \\
2 \\
4 \\
2\end{array}$ & $\begin{array}{l}\mathbf{y} \\
2 \\
4 \\
3\end{array}$ & $\begin{array}{l}y \\
2 \\
4 \\
4\end{array}$ & $\begin{array}{l}\mathbf{y} \\
\mathbf{2} \\
\mathbf{4} \\
5\end{array}$ & $\begin{array}{l}y \\
2 \\
4 \\
6\end{array}$ & $\begin{array}{l}\mathbf{y} \\
2 \\
4 \\
7\end{array}$ & $\begin{array}{l}\mathbf{y} \\
2 \\
4 \\
8\end{array}$ & $\begin{array}{l}\mathbf{y} \\
2 \\
4 \\
9\end{array}$ & $\begin{array}{l}\mathbf{y} \\
\mathbf{2} \\
5 \\
0\end{array}$ & $\begin{array}{l}\mathbf{y} \\
\mathbf{2} \\
\mathbf{5} \\
\mathbf{1}\end{array}$ & $\begin{array}{l}\mathbf{y} \\
2 \\
5 \\
2\end{array}$ & $\begin{array}{l}\mathbf{y} \\
2 \\
5 \\
3\end{array}$ & $\begin{array}{l}\mathbf{y} \\
\mathbf{2} \\
\mathbf{5} \\
\mathbf{4}\end{array}$ & $\begin{array}{l}y \\
2 \\
5 \\
5\end{array}$ & $\begin{array}{l}\mathbf{y} \\
\mathbf{2} \\
\mathbf{5} \\
\mathbf{6}\end{array}$ \\
\hline
\end{tabular}

Fig. 2(b) example for illustration of (16x16) pixel blocks

Fig. 2 shows a simple example for illustration of pixel blocks with $\mathbf{N}_{\mathbf{H}}=\mathbf{8}, \mathbf{N}_{\mathbf{V}}=\mathbf{8}$, and $\mathbf{B}=\mathbf{4}$.

The thick lines represent the block boundaries. In this example $\mathbf{N}_{\mathbf{H}_{\mathbf{B}}}=\mathbf{8}, \mathbf{N}_{\mathbf{H}_{\mathrm{B}}^{\mathrm{C}}}=\mathbf{4}, \mathbf{N}_{\mathbf{V}_{\mathrm{B}}}=\mathbf{8}$, and $\mathbf{N}_{\mathbf{V}_{\mathbf{B}}^{\mathrm{C}}}=\mathbf{4 8}$. 
The sets of pixel pairs in this example are

$$
\begin{aligned}
& \mathcal{H}_{B}=\{(y 4, y 5,)(y 12, y 13), \ldots \ldots(y 60, y 61)\} \\
& \mathcal{H}_{B}^{c}=\{(y 1, y 2),(y 2, y 3), \ldots .(y 63, y 64)\} \\
& \boldsymbol{v}_{B}=\{(y 25, y 33),(y 26, y 34), \ldots \ldots(y 32, y 40)\} \\
& \boldsymbol{v}_{B}^{C}=\{(y 1, y 9),(y 9, y 17),(y 17, y 25),(y 33, y 41), \ldots \ldots(y 56, y 64)\}
\end{aligned}
$$

Then we define the mean boundary pixel squared difference $\left(D_{B}\right)$ and the mean non boundary pixel squared difference $\left(D_{B_{C}}\right)$ for image y to be

$$
\begin{gathered}
\mathbf{D}_{\mathbf{B}}(\mathbf{y})=\frac{\sum_{\left(\mathbf{y}_{\mathrm{i}}, \mathbf{y}_{\mathbf{j}}\right) \in \mathbf{H}_{\mathbf{B}}}\left(\mathbf{y}_{\mathbf{i}}-\mathbf{y}_{\mathbf{j}}\right)^{2}+\sum_{\left(\mathbf{y}_{\mathrm{i}}, \mathbf{y}_{\mathbf{j}}\right) \in \mathbf{V}_{\mathbf{B}}}\left(\mathbf{y}_{\mathbf{i}}-\mathbf{y}_{\mathbf{j}}\right)^{2}}{\mathbf{N}_{\mathbf{H}_{\mathbf{B}}}+\mathbf{N}_{\mathbf{V}_{\mathbf{B}}}} \\
\mathbf{D}_{\mathbf{B}}^{\mathbf{C}}(\mathbf{y})=\frac{\sum_{\left(\mathbf{y}_{\mathbf{i}}, \mathbf{y}_{\mathbf{j}}\right) \in \mathbf{H}_{\mathbf{B}}}\left(\mathbf{y}_{\mathbf{i}}-\mathbf{y}_{\mathbf{j}}\right)^{2}+\sum_{\left(\mathbf{y}_{\mathrm{i}}, \mathbf{y}_{\mathbf{j}}\right) \in \mathbf{V}_{\mathbf{B}}}\left(\mathbf{y}_{\mathbf{i}}-\mathbf{y}_{\mathbf{j}}\right)^{2}}{\mathbf{N}_{\mathbf{H}_{\mathbf{B}}^{\mathrm{C}}}+\mathbf{N}_{\mathbf{V}_{\mathbf{B}}^{\mathrm{C}}}}
\end{gathered}
$$

Generally, as the quantization step size increases, $D_{B}$ will increase relative to $D_{B_{C}}$ ), and blocking artifacts will become more visible. Of course, this does not establish any level of correlation between (19), (20) and perceptual annoyance.

Also define the blocking effect factor

$$
\mathbf{B E F}(\mathbf{y})=\eta \cdot\left[\mathbf{D}_{\mathbf{B}}(\mathbf{y})-\mathbf{D}_{\mathbf{B}}^{\mathbf{C}}(\mathbf{y})\right]
$$

Where

$$
\eta= \begin{cases}\frac{\log _{2} B}{\log _{2}\left(\min \left(N_{H}, N_{V}\right)\right)} & \begin{array}{c}
\text { if } D_{B}(Y)>D_{B}^{C}(y) \\
\text { other wise }
\end{array}\end{cases}
$$

emphasizes the BEF as a function of block si2e. The assumption here is that the visibility of blocking effects increases with block size.

Of course, there can be multiple block sizes in a particular decoded image/video. For example, there can be 16X16 macro blocks and 4X4 transform blocks, both contributing to blocking effects.

Let $\boldsymbol{D}_{\boldsymbol{B}_{\boldsymbol{k}}}, \boldsymbol{D}_{\boldsymbol{B}_{\boldsymbol{k}}}^{\boldsymbol{C}}, \boldsymbol{B} \boldsymbol{E} \boldsymbol{F}_{\boldsymbol{k}}$, and $\eta_{k}$ modify (19)-(22) for block size . Then

$\mathbf{B E F}_{\mathbf{k}}(\mathbf{y})=\eta_{\mathbf{k}} \cdot\left[\mathbf{D}_{\mathbf{B}_{\mathbf{K}}(\mathbf{y})}-\mathbf{D}_{\mathbf{B}_{\mathbf{K}}}^{\mathbf{C}}(\mathbf{y})\right.$

The BEF over all block sizes is defined as

$$
\operatorname{BEF}_{\text {Tot }}(\mathbf{y})=\sum_{\mathrm{k}=1}^{\mathrm{K}} \operatorname{BEF}_{\mathbf{k}}(\mathrm{y})
$$

The mean-squared error including blocking effects (MSE-B) for reference image $\mathrm{x}$ and test image $\mathrm{y}$ is then defined as the sum of the MSE(x, y) in (1) and BEFtot (y) in (24)

$$
\begin{aligned}
& \text { MSE - B }(\mathbf{x}, \mathbf{y})=\mathbf{M S E}(\mathbf{X}, \mathbf{Y})+\mathbf{B E F}_{\text {Tot }}(\mathbf{y}) \\
& \text { Finally, we propose the PSNR-B as } \\
& \text { PSNR - B }(\mathbf{x}, \mathbf{y})=\mathbf{1 0 l o g}_{10} \frac{\mathbf{2 5 5 ^ { 2 }}}{\operatorname{MSE}-\mathbf{B}(\mathbf{x}, \mathbf{y})}
\end{aligned}
$$

The MSE term in (25) measures the distortion between the reference image and the test image, while the BEF term in (25) specifically measures the amount of blocking artifacts just using the test image. The BEF itself can be used as a no-reference quality index, these no-reference quality indices claim to be efficient for measuring the amount of blockiness, but may not be efficient for measuring image quality relative to fullreference quality assessment. On the other hand, the MSE is not specific to blocking effects, which can substantially affect subjective quality. We argue that the combination of MSE and BEF is an effective measurement for quality assessment considering both the distortions from the original image and the blocking effects in the test image. The associated quality index PSNR-B is obtained from the MSE-B by a logarithmic function, as is the PSNR from the MSE. The PSNR- B is attractive since it is specific for assessing image quality, specifically the severity of blocking artifacts.

\section{ALGORITHM FOR IMPLEMENTATION}

Step 1: start

Step2: read the input image (jpg, png, tif, bmp), name as I

Step 3: resize the image of dimension of $256 * 256$ in image I

Step 4: Construct the Compression for input image I using the DCT block coding and save the image as $I_{2}$ as compressed image and display the original image and compressed image

Step 5: compute the MSE, PSNR by using the equations (1)-(2) and calculate the MSE and PSNR values for Compressed Image $\mathrm{I}_{2}$ and display the values 
Step6: By using the Gaussian noise using k value compute the ssim_index by the equations (3)-(8) and display the values of mean and ssim for $\mathrm{I}_{2}$

Step 6: Compute the PSNR-B by using the equations (19)-(26) the PSNR-B is calculated for the image I2 and display the values

Step 7: Computing the MSE, PSNR, SSIM, PSNR-B are the quality metrics of the calculating image $\mathrm{x}$

De-blocked images:

Step 8: using low pass filter compute the image $g$ and display the image of the image $I_{2}$ named as $g$. and calculating the quality metrics for the image $\mathrm{g}$ and display the values

Pocs:

Step 9: By initialize the factor value $=1$ and delta_est $=[1: 2]$ compute the pocs named the image as $y$, resize the result image to $256 * 256$ and calculating the quality metrics for the image $y$ and display the values of $y$

Step10: Compute the median filter $(3 * 3)$ and $(7 * 7)$ for the image $I_{2}$ and display the results of $I_{2}$

Difference Images:

Step11: By using the equations (12)-(14) the difference images for $\mathrm{I}_{2}$, reshape and display the image $\mathrm{d}$

step12: repeat the step11 for $\mathrm{d} 1, \mathrm{~d} 2$ and display the images $\mathrm{d} 1, \mathrm{~d} 2$.

Step13: compute the performance analysis and plot the graphs

Step 14: stop.

\section{SIMULATION RESULTS ON DEBLOCKED IMAGES}

This section presents simulation results on quality assessment of deblocked images. Images are compressed using DCT block coding as JPEG. In JPEG, quantization is applied using a different quantization step size for each DCT coefficient, as defined by a quantization table. Here, we apply the same quantization step size for all DCT coefficients, to more directly investigate the effects of quantization step size on image quality. Quantization step sizes of 5, 10, 20, 40,80,120, and 160 were used in the simulations to investigate the effects of quantization step size. Deblocking was applied on the decoded images for comparison

\section{PSNR Analysis:}

Figure 3 shows that when the quantization step size was large $(\Delta \geq 80)$, the $3 \times 3$ filter, $7 \times 7$ filter and POCS methods resulted in higher PSNR than the no filter case on both the images. All the deblocking methods produced lower PSNR when the quantization step size was small $(\Delta \leq 30)$

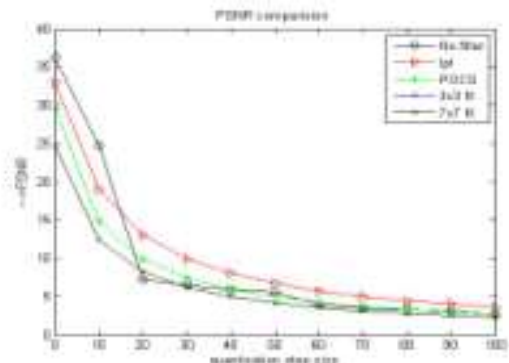

(a) Lena

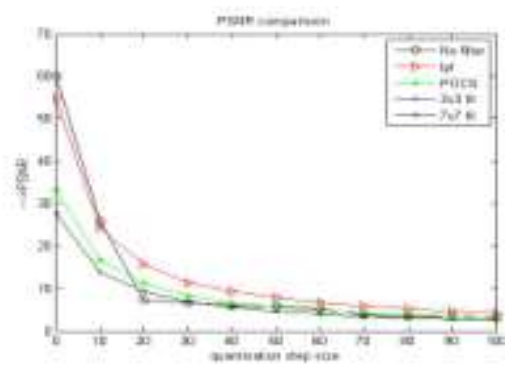

(c) Peppers

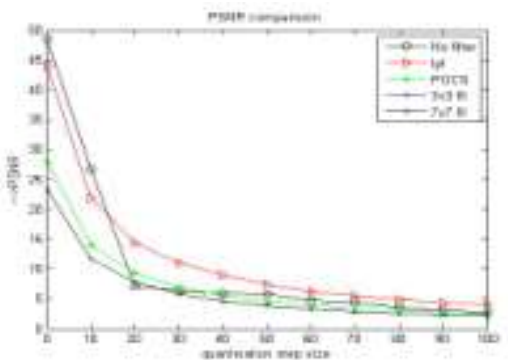

(b) Dewdrop

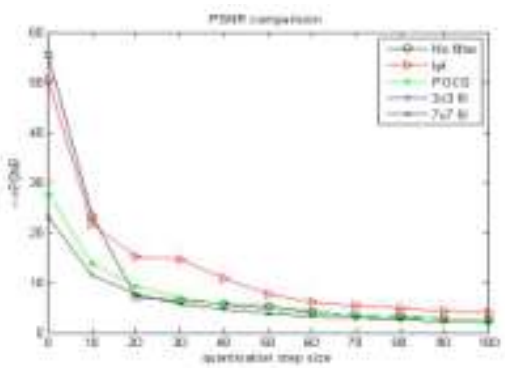

(d) Barbara

Fig 3. PSNR comparison of images (a) Lena. (b) Dewdrop, (c) Peppers. (d) Barbara

\section{SSIM Analysis:}

Figure 4 show that when the quantization step was large $(\Delta \geq 80)$, on the two images, all the filtered methods resulted in larger SSIM values. The $3 \times 3$ and $7 \times 7$ low pass filters resulted in lower SSIM values than the low filter case when the quantization step size was small $(\Delta \leq 30)$. 


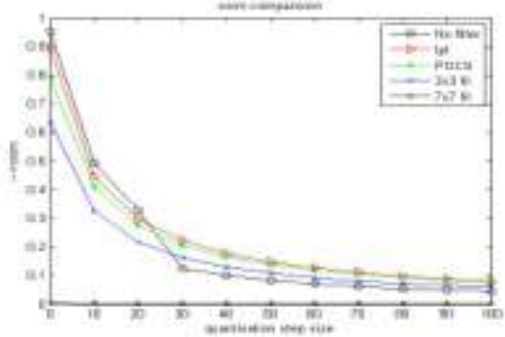

(a) Lena

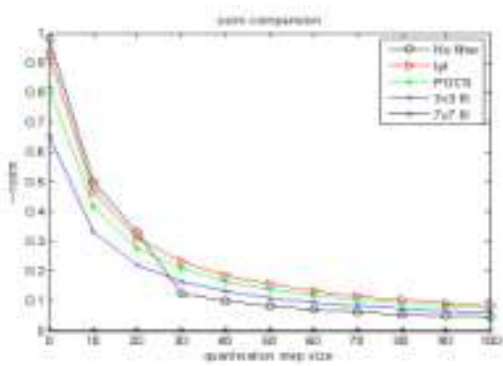

(c) Peppers

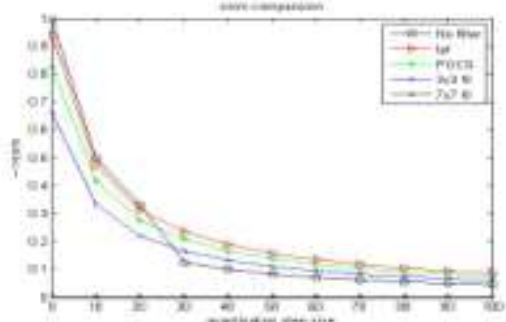

(b) Dewdrop

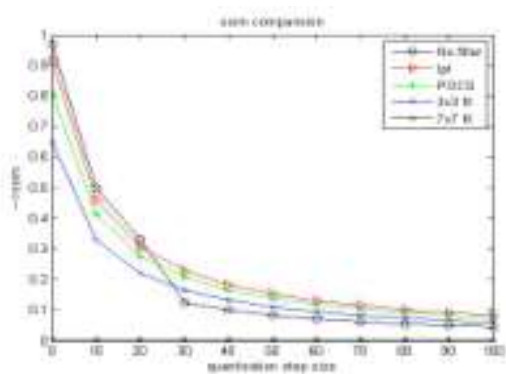

(d) Barbara

Fig 4. SSIM comparison of images (a) Lena. (b) Dewdrop, (c) Peppers. (d) Barbara

\section{E. PSNR-B Analysis:}

For large quantization steps, the PSNR-B values improved for the two images by employing low pass filtering methods. The POCS resulted in improved PSNR-B values compared to the no filtered case, even at small quantization step size.

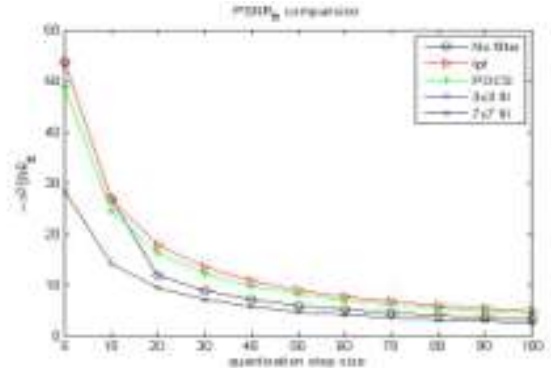

(a) Lena

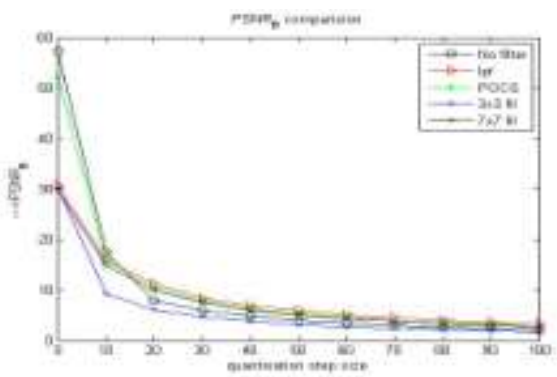

(c) Peppers

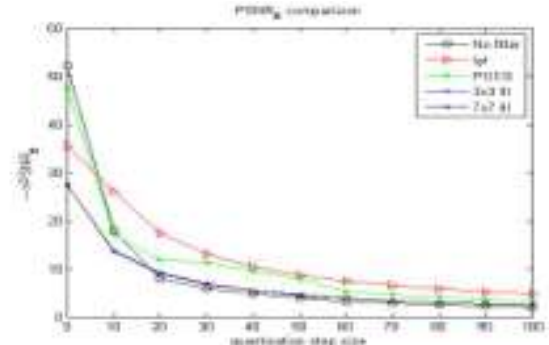

(b) Dewdrop

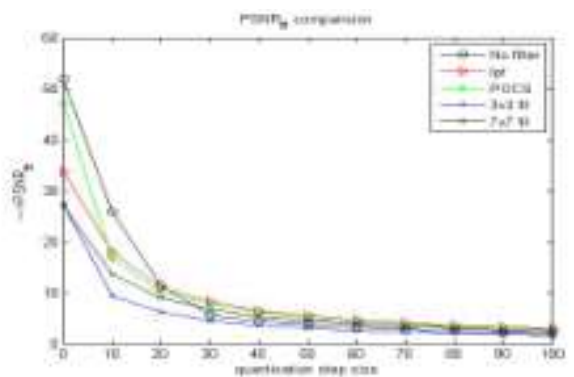

(d) Barbara

Fig 5. PSNR-B comparison of images (a) Lena. (b) Dewdrop, (c) Peppers. (d) Barbara 


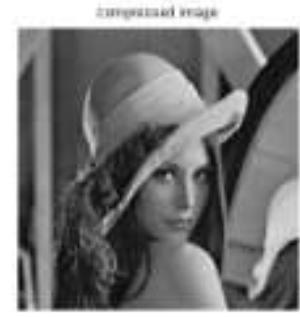

(a) no filter image

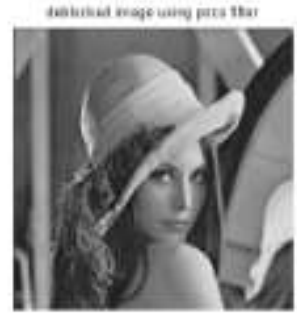

(b) POCS de blocking filter

Fig6. Reconstructed images of Lena with quantization step 80

(a) Quality metrics for no filter image: (Mean Square Error $=0.0780$, Peak Signal to Noise Ratio $=29.6041$ ssim_index=0.0099, PSNR_B=53.6695), (b) quality metrics for POCS de blocked filter image: (Mean Square Error $=0.0781$, Peak Signal to Noise Ratio $=29.6029$, ssim_index=0.0108, PSNR_B=53.6683).

Fig.6 shows Lena reconstructed from compression, also using quantization step 80. When no filter is applied as in Fig. 6(a), blocking artifacts are clearly visible, especially on the Lena. When the POCS deblocking filter was applied as in Fig. 6(b), the blocking effects were mostly removed, resulting in better subjective quality. The PSNR-B and SSIM quality indices produced larger values on the POCS filtered image, in agreement with observation.

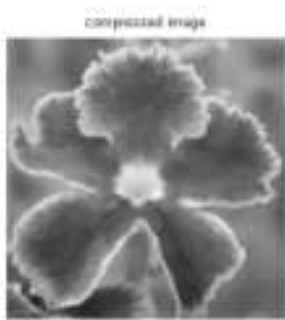

(a) no fillter image

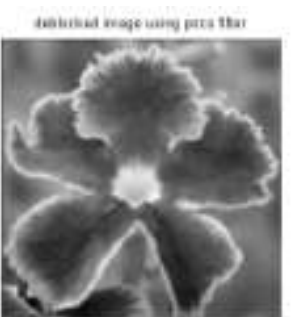

(b) POCS de blocking filter

Fig 7. Reconstructed images of Dewdrop with quantization step 80

(a) Quality metrics for no filter image: (Mean Square Error $=0.1600$, Peak Signal to Noise Ratio $=28.0454$ ssim_index=0.0069, PSNR_B=52.0070), (b) quality metrics for POCS de blocked filter image: (Mean Square Error $=0.1599$, Peak Signal to Noise Ratio $=28.0459$ ssim_index=0.0069, PSNR_B=52.1113) Fig. 7 shows Dewdrop reconstructed from compression, also using quantization step 80. When no filter is applied as in Fig. 7(a), blocking artifacts are clearly visible, especially on the leaves. When the POCS deblocking filter was applied as in Fig. 7(b), the blocking effects were mostly removed, resulting in better subjective quality. The PSNR-B and SSIM quality indices produced larger values on the POCS filtered image, in agreement with observation

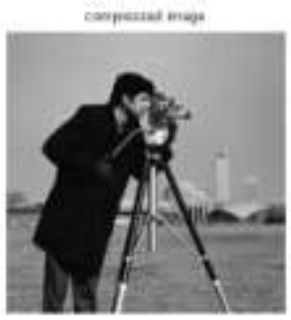

(a) no filter image

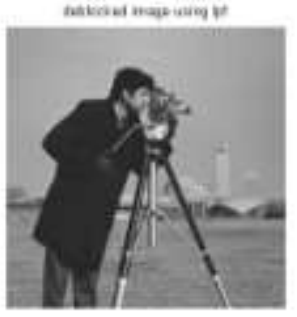

(b) LPF de blocking filter

Fig 8. Reconstructed images of Cameraman with quantization step 80

(a) Quality metrics for no filter image: (Mean Square Error $=0.1933$, Peak Signal to Noise Ratio $=27.6343$ ssim_index=0.0120, PSNR_B=51.4032), (b) quality metrics for POCS de blocked filter image: (Mean Square Error $=0.1933$, Peak Signal to Noise Ratio $=27.6346$, ssim_index=0.0120, PSNR_B=51.4340). 
Fig. 8 shows Cameraman reconstructed from compression, also using quantization step 80 . When no filter is applied as in Fig. 8(a), blocking artifacts are clearly visible, especially on the Cameraman. When the LPF deblocking filter was applied as in Fig. 8(b), the blocking effects were greatly removed, resulting in better subjective quality. The PSNR-B and SSIM quality indices produced larger values on the POCS filtered image, in agreement with observation.

\section{CONCLUSION}

We have tested our algorithm on few natural images. Those sample images are shown in above figure. We have found that the better quality metric is obtained at quality factor 70 for JPEG compression. This Analysis will brings out a new trend in the quality metrics of the image and proves to be efficient than the conversional metrics.

\section{References}

[1] S.S Channappayya, A.C.Bovik, and R.W.Heath, Jr, "Design of linear equalizers optimized for the structural similarity index," IEEE Trans. Image Process., vol.17, no.6, pp.857-872, Jun.2008.

[2] Z.Wang, A.C.Bovik, H.R.Sheikh, and E.P.Simoncelli, "Image quality assessment: From error visibility to structural similarity," IEEE Trans. Image Process., vol.13, no.4, pp.600-612, Apr.2004.

[3] A.Zakhor, "Iterative procedure for reduction of blocking effects in transform image coding,” IEEE Trans. Circuits Syst. Video Technol., vol.2,no.1, pp.91-95,Mar.1992.

[4] .Yang, N.P.Galatsanos, and A.K.Katsaggelos, "Projection-based spatially adaptive reconstruction of block- transform compressed images, ” IEEE Trans. Image Process., vol.4, no.7, pp.896-908, Jul.1995.

[5] Y.Yang, N.P.Galatsanos, and A.K.Katsaggelos, "Regularized reconstruction to reduce blocking artifacts of block discrete cosine transform compressed images,” IEEE Trans. Circuits Syst. Video Technol., vol.3, no.6, pp.421-432, Dec.1993.

[6] H.Paek, R.C.Kim, and S.U.Lee, "On the POCS-based post processing technique to reduce the blocking artifacts in transform coded images, ” IEEE Trans. Circuits Syst. Video Technol., vol.8, no.3, pp.358-367,Jun.1998.

[7] S.H.Park, and D.S.Kim, “Theory of projection onto narrow quantization constraint set and its applications," IEEE Trans. Image Process., vol.8, no.10, pp.1361-1373, Oct.1999.

[8] Z.Wang, A.C.Bovik, and E.P.Simoncelli, "Multi-scale structural similarity for image quality assessment," in Proc. IEEE Asilomar Conf.Signal Syst. Comput.,No v.2003.

[9] A.C.Bovik, and H.R.Sheikh,"Image information and visual quality," IEEE Trans. Image Process., vol.15, no.2, pp.430-444, Feb.2006.

[10] A.C.Bovik, and H.R.Sheikh, "A statistical evaluation of recent full reference image quality assessment algorithms,” IEEE Trans. Image Process., vol.15, no.11, pp.3441-3452, Nov.2006.

[11] Z.Wang, and A.C.Bovik, “A Universal image quality Index, ” IEEE Trans. Signal Process.Lett., vol.9, no.3, pp.81-84, Mar.2002.

[12] S.Liu, and A.C.Bovik, "Efficient DCT-domain blind measurement and reduction of blocking artifacts,” IEEE Trans. Circuits Syst Video Technol., vol.12, no.12, pp.1139-1149, Dec.2002.

[13] B.Girod, “What's wrong with mean-squared error," in Digital images and human vision, A.B.Watson, ED. Cambridge, MA: MIT Press, 1993, pp.207-220.

[14] Z.Wang, and A.C.Bovik," Blind measurement of blocking artifacts in images, ”in Proc.IEEE Int.Conf.Image Process., Vancouver, Canada, Oct.2000.pp.981-984.

[15] Y.Jeong, I.Kim, and H.Kang," Practical projection based postprocessing of block coded images with fast convergence rate,” IEEE Trans. Circuits Syt. Video Technol., vol.10, no.4, pp.617-623, Jun.2000.

\section{ABOUT AUTHORS PROFILE}
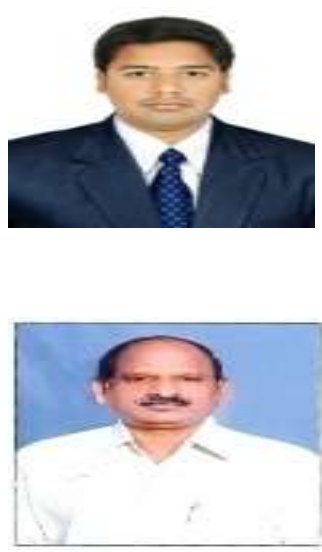

Trinadh Tadala graduated from Lenora college of Engineering, Rampachodavaram in Electronics And Communication Engineering (ECE) Stream From JNTUK, Now pursuing Masters in Computers And Communication (C\&C) Stream from University college of Engineering, JNTUK, Kakinada, Andhra Pradesh, India.

Sri E.Venkata Narayana completed B.E and M.E in first class from Andhra University, Visakhapatnam. He has been in teaching since 1985. Presently he is working as Asst. Professor in Dept. of ECE, JNTU college of Engineering Kakinada. He published more than 45 papers in national and international conferences and journals. 